\title{
Downregulation of miRNA-451a Promotes the Differentiation of CD4+ T Cells towards Th2 Cells by Upregulating ETS1 in Childhood Asthma
}

\author{
Tianyue Wang Qianlan Zhou Yunxiao Shang \\ Department of Pediatrics, Shengjing Hospital of China Medical University, Shenyang, PR China
}

\section{Keywords}

miRNA-451a - ETS proto-oncogene 1 . Childhood asthma .

Th2 cell differentiation · Type 2 inflammation

\begin{abstract}
Children exposed to common aeroallergens may develop asthma that progresses into adulthood. Inflammation regulated by T helper 2 (Th2) cells, a specific subpopulation of CD4+ T lymphocytes, is involved in asthmatic injury. Herein, our microarray data indicated that microRNA-451a-5p (miRNA-451a) expression decreased by 4.6 -fold and ETS protooncogene 1 (ETS1) increased by 2.2-fold in the peripheral blood lymphocytes isolated from asthmatic children $(n=4)$ as compared to control individuals $(n=4)$. The negative correlation between miRNA-451 $a$ and ETS1 was further validated in 40 CD4+ T cell samples ( 10 healthy vs. 30 asthmatic samples). In vitro, naïve CD4+ T cells isolated from control individuals were cultured under Th2 cell polarizing condition. miRNA-451a expression decreased while ETS1 increased in CD4+ T cells in the setting of Th2 cell polarization. Moreover, miRNA-451a knockdown enhanced Th2 cell polarization - cells positive for both GATA3 (GATA binding protein 3, a Th2-transcription factor) and CD4 increased, and the generation of Th2 cell cytokines, interleukin (IL)5 and IL13, increased. In contrast, miRNA-451a overexpression inhibited
\end{abstract}

karger@karger.com www.karger.com/jin

Karger"

BOPEN ACCESS
(C) 2020 The Author(s)

Published by S. Karger AG, Basel

This article is licensed under the Creative Commons AttributionNonCommercial-NoDerivatives 4.0 International License (CC BYNC-ND) (http://www.karger.com/Services/OpenAccessLicense) Usage and distribution for commercial purposes as well as any distribution of modified material requires written permission.
Th2 cell differentiation. Interestingly, dual-Luciferase assay proved ETS1 as a novel target of miRNA-451a. Moreover, enforced expression of ETS1 partially restored miRNA-451ainduced inhibition of IL5 and IL13, and increased the GATA3+CD4+ cell population. Collectively, our work demonstrates that downregulation of miRNA-451a upregulates ETS1 expression in CD4+ T cells, which may contribute to Th2 cell differentiation in pediatric asthma.

(C) 2020 The Author(s)

Published by S. Karger AG, Basel

\section{Introduction}

As a common chronic immunological disease, asthma affects people from childhood to adulthood [1]. Children exposed to common aeroallergens may develop asthma that progresses into adulthood $[2,3]$. Such clinical findings highlight the urgent need to identify the underlying mechanisms of asthma that occurs in early life. Sensitization of the airways to aeroallergens commonly results from selective expansion of T lymphocytes, particularly $\mathrm{T}$ helper 2 (Th2) cells $[4,5]$. Th2 cells, a specific subpopulation of $\mathrm{CD} 4+\mathrm{T}$ cells, are known to generate a group of type 2 cytokines, including interleukin (IL)4, IL5, and IL13, to orchestrate the asthma-associated airway granu- 
locyte infiltration, excess mucus production, airway remodeling, and hyper-reactivity [6]. IL4 and IL5 is able to facilitate eosinophil recruitment and B-cell class switching, establishing a plausible link between $\mathrm{T}$ cells producing these cytokines and allergic disease [7]. Recent studies have discovered that type 2 innate lymphoid cells (ILC2s) acting similarly to Th2 cells are critical effectors of the type 2 immune response to allergens and implicated in asthma $[8,9]$. Considering asthma as a disease resulting from type 2 inflammation, lymphocytes ( $\mathrm{T}$ cells and ILC2s) have been recognized as contributors to asthma pathogenesis [7]. Glucocorticoids with the capability of suppressing the type 2 inflammatory response are the most effective drugs used in treating asthma $[1,10]$. However, a high dose and prolonged use of these drugs leads to varied local and systemic side effects [1]. More specific inhibitors of type 2 inflammation, such as anti-IL5 antibody, have been introduced into the clinic to treat asthma [11]. Understanding the molecular mechanisms underlying the type 2 inflammation in the setting of asthma will help to develop novel therapeutic strategies for childhood asthma.

As endogenous small RNA molecules, the microRNAs (miRNAs) are identified as regulators of post-transcriptional gene silencing and control the expression of approximately $30 \%$ of all genes [12]. There is an increasing attention on miRNAs because of their functional effect on polarization of CD4+ T cells [13-15]. Intensive effort has been devoted to identifying the miRNA-gene axes involved in asthma-associated type 2 inflammation.

In this study, data mining from the present miRNA and mRNA microarray results showed that the expression of miRNA-451a-5p (referred to miRNA-451a) markedly decreased in lymphocytes from children with asthma. We also noted that the expression of miRNA-451a was negatively correlated with that of ETS proto-oncogene 1 (ETS1), a predictive target of miRNA-451a. Although a previous study revealed that downregulation of miRNA-451 a contributes to the activation and proliferation of CD4+ T cells [16], its role in Th cell polarization in pediatric asthma is unknown. ETS1 belongs to the ETS family of transcription factors and possesses a conserved ETS DNA binding domain that recognizes the core consensus DNA sequence GGAA/T present in target genes [17]. Interestingly, loss of ETS1 impaired the generation of Th2 cytokines, IL5 and IL13, in ILC2s, another cell population that regulates type 2 allergic inflammation $[6$, 18]. This study suggests a requirement of ETS1 expression for the differentiation of type 2 inflammation-related immune cells. A hypothesis that elevation of ETS1 con-
Table 1. Clinical characteristics

\begin{tabular}{lllc}
\hline & Controls & $\begin{array}{l}\text { Asthmatic } \\
\text { children }\end{array}$ & $\begin{array}{l}p \text { value } \\
\text { (Student } \\
t \text { test) }\end{array}$ \\
\hline Female, $n$ & 1 & 1 & - \\
Male, $n$ & 3 & 3 & - \\
Recurrent wheezing & no & yes & 0.89 \\
Age, years & $6.5 \pm 2.4$ & $6.8 \pm 2.4$ & 0.41 \\
Height, cm & $120.5 \pm 14.1$ & $128.8 \pm 12.5$ & $<0.001$ \\
FEV,$\%\left(\beta_{2}\right.$ agonist) & $0.14 \pm 1.66$ & $14.7 \pm 2.5$ & 0.001 \\
Serum IgE, IU/mL & $26.9 \pm 10.3$ & $219.2 \pm 63.4$ & 0.001 \\
\hline
\end{tabular}

Data are presented as the mean $\pm \mathrm{SD}$, or as indicated. $p<0.05$ was considered significant.

tributes to Th2 cell polarization in pediatric asthma is proposed. Whether ETS1 is regulated by miRNA-451a requires further elucidation.

In this study, expanded CD4+ T cell samples were isolated from children with asthma and controls, and the correlation between miRNA-451a and ETS1 expression was determined. Moreover, miRNA-451a gain- and lossof-function experiments were performed to study its role in Th2 cell differentiation in vitro.

\section{Materials and Methods}

Human Samples

According to recommendations of the Global Initiative for Asthma (GINA), asthmatic children whose forced expiratory volume in $1 \mathrm{~s}\left(\mathrm{FEV}_{1}\right)$ increased by $12 \%$ following the inhalation of $\beta_{2}$ agonist were recruited in Shengjing Hospital of China Medical University, and their clinical details are summarized in Table 1.

\section{Microarrays}

A blood sample $(4-6 \mathrm{~mL})$ from each participant was mixed with lymphocyte separation medium (P8610, Solarbio, Beijing, China) to isolate lymphocytes via centrifugation $(1,000 \mathrm{~g}, 30 \mathrm{~min}$, room temperature). Lymphocytes lay between the plasma layer and separation medium. The purity of lymphocytes was confirmed with Wright-Giemsa stain. Total RNAs were extracted from the lymphocytes $\left(2 \times 10^{6}\right.$ per sample) collected from 4 asthmatic and 4 healthy children. The miRNA and mRNA expression profiles were obtained via Agilent human miRNA microarray containing probes for 2,549 mature miRNAs (ID 070156) and gene microarray containing probes for 18,463 genes (ID 084410; Agilent Tech, Santa Clara, CA, USA). In brief, RNAs were quantified via NanoDrop ND-2000 (Thermo Scientific, Pittsburgh, PA, USA) and their integrity was tested with Bioanalyzer 2100 (Agilent Tech). A sample of RNA integrity number $\geq 7$ and $28 S / 18 S \geq 0.7$ was used as the template. One sample was divided into two parts for miRNA and mRNA microarrays. Sample labeling and microarray hybrid- 
ization were performed at Shanghai OE Biotech Co. Ltd (Shanghai, China). Raw data were scanned with a Scanner G2505C (Agilent Tech) and exported via Feature Extraction software (Agilent Tech). The Student $t$ test was used to analyze the difference between the two groups. A fold change (FC) $\geq 2$ and $p$ value $<0.05$ were used to select differentially expressed miRNAs and mRNAs. A hierarchical clustering heatmap was used to show the expression patterns of miRNAs and genes among samples. The normalized signal intensities were analyzed and calculated with the Pearson correlation coefficient (PCC) to evaluate the correlation between miRNAs and genes. A miRNA-gene pair with PCC $r \leq-0.7$ ( $p$ value $<0.05$ ) was considered a significant correlation.

\section{Culture of CD4+ Cells and Th2 Cell Differentiation}

Lymphocytes collected from mixed blood samples from 4 healthy children were subjected to isolation of CD4+ cells. Briefly, lymphocytes $\left(1 \times 10^{7}\right)$ suspended in an $80-\mu \mathrm{L}$ buffer were incubated with 20 $\mu \mathrm{L}$ of CD4 microbeads (130-045-101; Miltenyi Biotec, Bergisch Gladbach, Germany) for $15 \mathrm{~min}$ at $4^{\circ} \mathrm{C}$. After washing, the cell-bead mixture was centrifuged at $300 \mathrm{~g}$ for $10 \mathrm{~min}$ and cell pellets were collected and then separated via MACS separator according to the manufacturer's protocols. The purity of CD4+ cells was over $95 \%$ as confirmed with flow cytometry assay (online suppl. Fig. s1; for all online suppl. material, see www.karger.com/doi/10.1159/000509714). The demographic and clinical characteristics of all participants are included in online supplementary Table s1.

The isolated CD4+ cells were added to 24-well plates coated with anti-CD3 and anti-CD28 antibodies (16-0037-81 and 160289-81, eBioscience, Shanghai, China). Cells were first cultured in RPMI-1640 (Gibco, Grand Island, NY, USA) supplemented with $30 \mathrm{IU} / \mathrm{mL}$ IL2 (11848-HNAE, Sino Biological Inc., Beijing, China) and $10 \%$ fetal calf serum for $48 \mathrm{~h}$, and then replaced by the medium with $100 \mathrm{IU} / \mathrm{mL}$ IL2 for approximately 14 days to allow cell expansion (purity $>90 \%$ determined with flow cytometry). Thereafter, the cells were maintained in RPMI-1640 supplied with $1 \mu \mathrm{g} / \mathrm{mL}$ anti-CD3 and $2 \mu \mathrm{g} / \mathrm{mL}$ anti-CD28 antibodies (eBioscience).

To induce Th2 cell differentiation, naïve CD4+ T cells were incubated with $10 \mathrm{ng} / \mathrm{mL}$ of IL 4 and $10 \mu \mathrm{g} / \mathrm{mL}$ of anti-IFN- $\gamma$ for $96 \mathrm{~h}$. Thereafter, these cells were treated with $50 \mathrm{ng} / \mathrm{mL}$ of phorbol12-myristate-13-acetate (PMA; MCE, Monmouth Junction, NJ, USA) and $2 \mu \mathrm{m}$ of ionomycin (Aladdin, Shanghai, China) for an additional $6 \mathrm{~h}$.

\section{Flow Cytometry}

CD4+ cells $\left(5 \times 10^{5}\right)$ suspended in $100 \mu \mathrm{L}$ of PBS were incubated with $2 \mu \mathrm{L}$ of anti-CD4 FITC antibody (130-113-775; Miltenyi Biotec) and/or $2 \mu \mathrm{L}$ of anti-GATA3 PE antibody (130-109-161, Miltenyi Biotec) in the dark at $4{ }^{\circ} \mathrm{C}$ for $10 \mathrm{~min}$. Cells were washed with $1 \mathrm{~mL}$ of buffer, centrifuged at $300 \mathrm{~g}$ for $10 \mathrm{~min}$, and then analyzed with flow cytometer (NovoCyte, San Diego, CA, USA).

\section{Lentivirus Infection}

miRNA-451a (MIMAT0001631: 5' -aaaccguuaccauuacugaguu3'), miR-3646 (MIMAT0018065: 5' -aaaaugaaaugagcccagccca-3'), and ETS1 (NM_005238) overexpression, and miRNA-451a and miR-3646 knockdown in CD4+ T cells were mediated by lentivirus systems: TET-PLKO-PURO (Addgene, Cambridge, MA, USA) for miRNA-451a and miR-3646, and PLJM1-FLAG (FenfhuiBio, Changsha, China) for ETS1. High-titer lentiviruses $\left(10^{8} \mathrm{TU} / \mathrm{mL}\right)$, including LV-miRNA-451a, LV-anti-miRNA-451a, LV-mi-
Table 2. Real-time qPCR primers

\begin{tabular}{ll}
\hline Name & $5^{\prime}-3^{\prime}$ \\
\hline IL5 F & atctttcagggaataggc \\
IL5 R & aagaaactcttgcaggtag \\
IL13 F & agatggtatggagcatcaa \\
IL13 R & tctgacccagatgaggtg \\
ETS1 F & tttgtcgtaatagtagcgtag \\
ETS1 R & ggggaggaagtaggaagagt \\
CDH11 F & gtgtttcatagtccgttgtg \\
CDH11 R & tttatctgggtgtctcgc \\
MYO3A F & ttccctgactgtctttcc \\
MYO3A R & ccaggcttacctcactaacg \\
PABPC3 F & ggttctgagtctgtgggaca \\
PABPC3 R & atggattttggagcagggt \\
miRNA-1246 F & gcagggtccgaggtattc \\
miRNA-1246 R & ggattttggagcagggtt \\
miRNA-3646 F & gcagggtccgaggtattc \\
miRNA-3646 R & cgtttggtcccttcaacc \\
miRNA-133b F & gcagggtccgaggtattc \\
miRNA-133b R & ggggctgcgcgaggcagt \\
miRNA-4449 F & gcagggtccgaggtattc \\
miRNA-4449 R & aaaccgttaccattactgagtt \\
miRNA-451a F & gtgcagggtccgaggtattc \\
miRNA-451a R & \\
\hline
\end{tabular}

RNA-3646, LV-anti-miRNA-3646, LV-ETS1 overexpression, and negative controls (NC) were obtained according to the suppliers' instructions. CD $4+\mathrm{T}$ cells $\left(5 \times 10^{5}\right.$ cells $)$ seeded in 6 -well plates were maintained in Th2 differentiation media for $24 \mathrm{~h}$, and then infected with viruses at an MOI of 20. Seventy-two hours later, cells were stimulated with PMA and ionomycin for an additional $6 \mathrm{~h}$.

\section{PmirGLO Dual-Luciferase Assay}

There were 2 potential binding sites on ETS1 for miRNA-451a as predicted via the mirnada database. The wild-type (WT-1 and WT-2) fragments and mutants (MT-1 and MT-2) were inserted into pmirGLO dual-luciferase expression vector. PmirGLO plasmids and miRNA-451a/NC mimics were transfected into $293 \mathrm{~T}$ cells. Normalized firefly luciferase activity (firefly/Renilla luciferase activity) was compared to that of cells transfected with pmirGLO-WT and NC mimics. The luciferase activity was averaged from 3 replicates.

\section{Western Blot Assay}

Protein fractions were extracted from $1 \times 10^{6}$ cells with RIPA Lysis Buffer (SolarBio, Beijing, China), and quantified with a BCA kit (Beyotime, Shanghai, China). Proteins (15-20 $\mu \mathrm{g})$ were separated on SDS-PAGE before transferring onto PVDF membranes (Millipore, Billerica, MA, USA). Membranes were incubated with $5 \%(\mathrm{M} / \mathrm{V})$ non-fat milk for $60 \mathrm{~min}$, and incubated overnight at $4{ }^{\circ} \mathrm{C}$ with antibodies (all from ABclonal, Shanghai, China) against ETS1 (A13302, 1:1,000), IL5 (A7690, 1:1,000), and IL13 (A2089, 1:500), followed by incubation with IgG-HRP for $60 \mathrm{~min}$. After visualization, blot intensities were analyzed with Gel Pro-analyzer. GAPDH (60004-1-Ig, 1:10,000; ProteinTech, Rosemont, IL, USA) was used as a control.
40

J Innate Immun 2021;13:38-48

DOI: $10.1159 / 000509714$
Wang/Zhou/Shang 
Quantitative Real-Time PCR

Cellular RNAs were isolated via an RNAsimple extraction kit (TIANGEN Biotech, Beijing, China), quantified via NanoDrop ND-2000, and reversely processed into cDNAs via a reverse transcription (RT) primer for mRNA expression detection, and via a loop primer $\left(5^{\prime}\right.$-gttggctctggtgcagggtccgaggtattcgcaccagagccaacaactca- $3^{\prime}$ ) for miRNA-451a expression detection. Real-time qPCR was carried out on an Exicycler 96 sequence detection system (Bioneer, Daejeon, Korea) according to the manufacturer's protocols. The mRNA expression was normalized to GAPDH, while the miRNA-451a expression was normalized to U6. The primers for real-time qPCR are listed in Table 2.

\section{Enzyme-Linked Immunosorbent Assay}

Cell supernatants were collected and diluted (1:2 for IL5 and 1: 5 for IL13). The contents of IL5 and IL13 in cell supernatant were determined with enzyme-linked immunosorbent assay (ELISA) kits (LIANKE Biotech Co. Ltd, Hangzhou, China) according to the manufacturer's protocols.

\section{Statistics}

One-way ANOVA followed by Bonferroni's multiple comparison test was used to analyze data from multiple groups. The Student $t$ test was used to analyze data between the two groups. $p$ values $<0.05$ were considered statistically significant.

\section{Results}

Downregulation of miRNA-451a and Upregulation of ETS1 Were Observed in Lymphocytes Derived from

\section{Children with Asthma}

The population characteristics of age- and sexmatched asthmatic and healthy children are shown in Table 1 . The average $\mathrm{FEV}_{1}$ value ( $\beta_{2}$ agonist) and serum IgE value of asthma patients were $14.7 \%$ and $219.2 \mathrm{IU} /$ $\mathrm{mL}$, which were significantly higher than those of the control individuals. Lymphocytes were extracted from the whole blood of each participant and analyzed with miRNA and mRNA microarrays. miRNAs and mRNAs with FC $\geq 2$ and $p<0.05$ were considered differentially expressed. Two miRNAs were upregulated and three were downregulated in asthmatic lymphocytes (Fig. 1a, b). miRNA-451a with an FC of 4.6, and a $p$ value of 0.008 (asthma vs. control) was selected for the following study due to its regulatory role in the activation and proliferation of CD4+ T cells [16]. Real-time qPCR was performed to validate the expression of differentially expressed miRNAs and genes using RNA samples subjected to microarray analysis as templates (online suppl. Fig. s2A, B).

The expression of miRNA-451a was further analyzed in expanded CD4+ T cell samples ( 10 healthy and 30 asthmatic children). Our data demonstrated that miRNA- 451a was downregulated in CD4+ T cells derived from children with asthma (online suppl. Fig. s2C).

Moreover, 355 mRNAs were upregulated and 346 were downregulated in asthmatic lymphocytes as compared to control cells (Fig. 1c, d). The correlation between miRNA-451a and differentially expressed mRNAs was calculated with PCC based on microarray data. mRNAs with a negative correlation to miRNA-451a were screened according to a PCC $r$ value $<-0.7$ and $p$ value $<0.05$. A total of 197 mRNAs were identified (Fig. 1e). In addition, the potential targets of miRNA-451a were predicted with miranda software. Targets with a score over 140 (58 genes) were cross-checked with the above 197 mRNAs, and 29 genes were screened out, including ETS1 (Fig. 1e; online suppl. Table s2).

Results from real-time qPCR showed that ETS1 significantly increased in CD4+ T cells from children with asthma (online suppl. Fig. s2D). We also analyzed the correlation between miRNA-451a and ETS1 in 10 healthy and 30 asthmatic samples, and found a negative correlation between their expression (Fig. 1f; $r=-0.4$, $p=0.03)$.

\section{miRNA-451a Overexpression Inhibits the}

Differentiation of CD4+ T Cells towards Th2 Cells

Naïve CD4+ T cells were maintained in Th2 differentiation culture media for $96 \mathrm{~h}$. Cells positive for both GATA3 (GATA binding protein 3, a Th2 transcription factor) and CD4 were analyzed with flow cytometry assay (Fig. 2a). Moreover, cells were double stained with GATA3 and CD4 in the setting of Th2 differentiation induction. The expression levels of Th2 cell cytokines (IL5 and IL13) and miRNA-451a were detected with real-time qPCR. A significant elevation in IL5 and IL13 also indicated differentiation towards Th2 post-induction (Fig. 2b). Interestingly, the expression of miRNA-451a decreased and that of ETS1 increased in response to Th2 polarization (Fig. 2c).

Fig. 1. Downregulation of miRNA-451a and upregulation of ETS1 observed in lymphocytes derived from children with asthma (CA). a, c Volcano blots showed downregulated miRNAs as blue dots, and upregulated miRNAs as red dots. b, d All differentially expressed miRNAs and mRNAs are shown in heatmaps. Each row represents an miRNA or an mRNA, and each column indicates a sample. e The predictive targets of miR-451a with expression levels negatively correlated with miR-451a based on microarray data are shown in the Venn diagram. $\mathbf{f}$ The expression correlation between miR-451a and ETS1 CD4+ T cell samples from 10 control and 30 asthmatic children was analyzed with the Spearman correlation test $(p<0.05)$. 

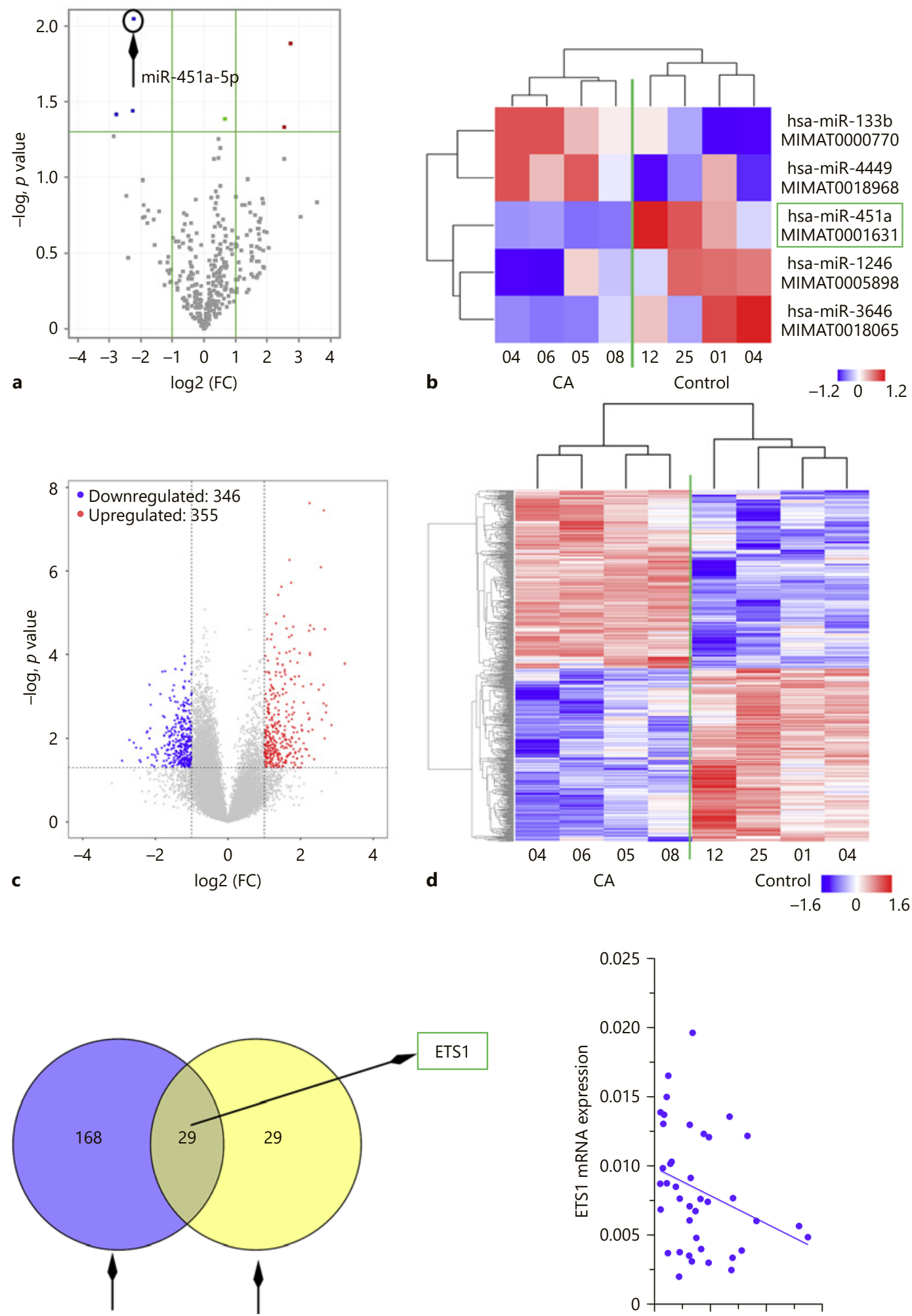

Correlation $<-0.7 \quad$ Predicted by

e $\quad p$ value $<0.05$ mirnada database

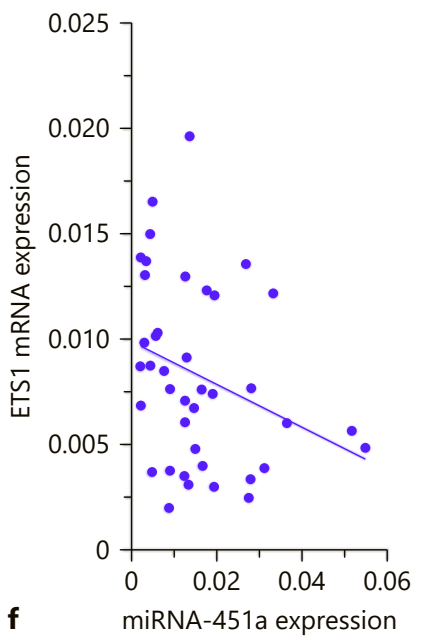



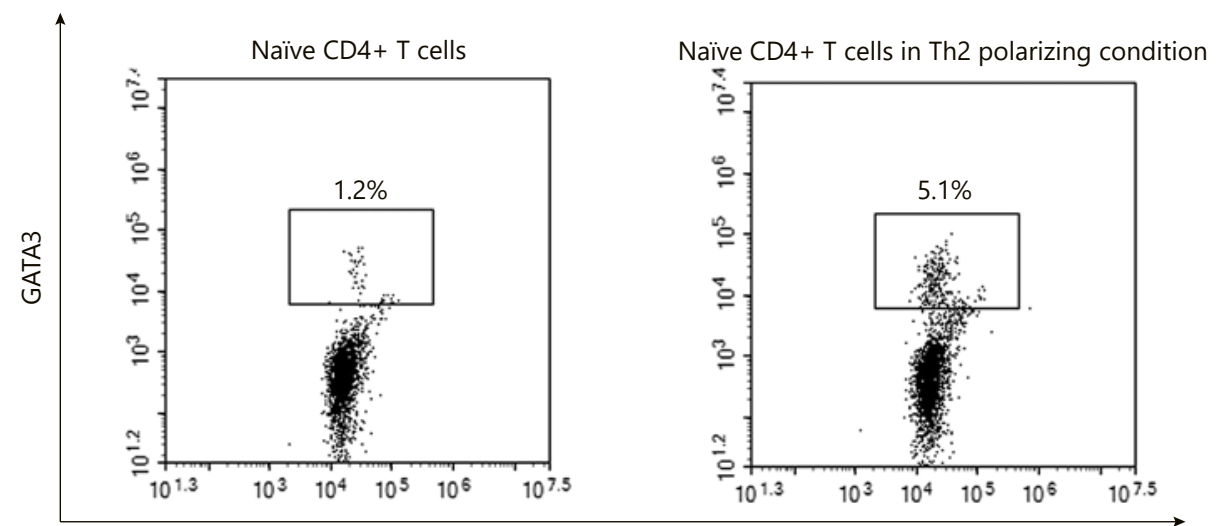

a

CD4
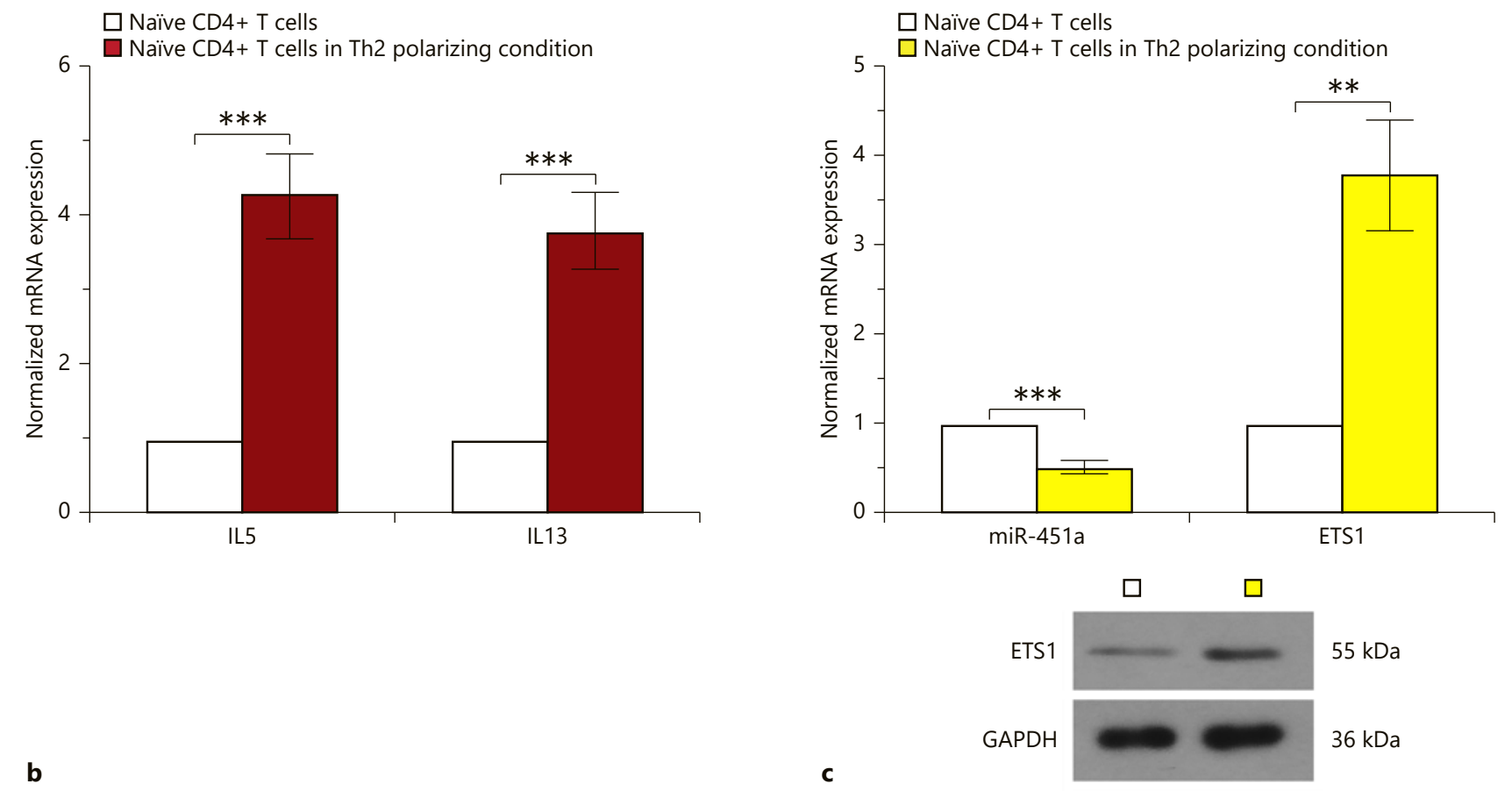

Fig. 2. Downregulation of miRNA-451a and upregulation of ETS1 are observed during the differentiation of CD4+ T cells towards Th2 cells. a Representative images of flow cytometry analysis showing GATA3+CD4+ cells. b qRT-PCR analysis of IL5 and IL13

Anti-miRNA-451a lentiviruses were used to block the expression and function of endogenous miRNA-451a in CD4+ $\mathrm{T}$ cells cultured in Th2 differentiation medium (online suppl. Fig. s3A, B). Silencing of miRNA-451a further upregulated the GATA3+CD4+ cell percentage and augmented the production of IL5 and IL13 induced by Th2 differentiation medium (Fig. 3a, b, e). In contrast, overexpression of miRNA-451a displayed opposite ef-

in naïve CD4+ T cells under Th2 polarizing conditions. c The level of miRNA-451a and ETS1 was analyzed by qRT-PCR or Western blot. Statistically significant differences in $\mathbf{b}$ and $\mathbf{c}$ were analyzed by two-tailed unpaired $t$ test $(n=3) .{ }^{* *} p<0.01,{ }^{* * *} p<0.001$.

fects (Fig. 3c, d, f). These data suggested that miRNA451a downregulation was required for Th2 cell differentiation.

Aside from miRNA-451a, miRNA-3646 had an FC of $4.8(p=0.04)$. We additionally explored its role in regulating Th2 cell differentiation by gain- and loss-of-function experiments. The efficiency of lentivirus-mediated overexpression or knockdown of miR-3646 was determined 


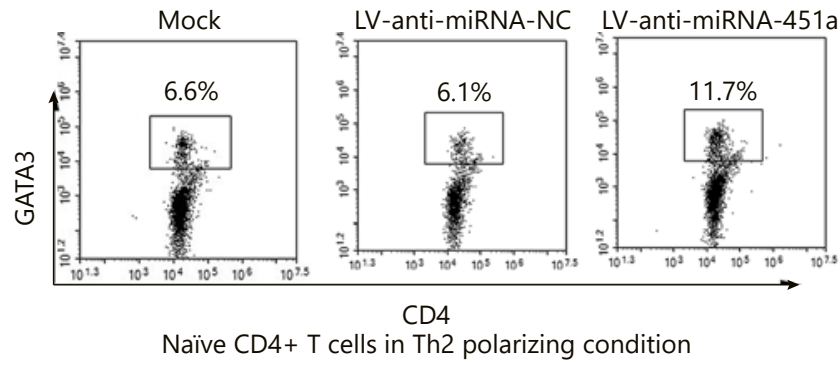

a

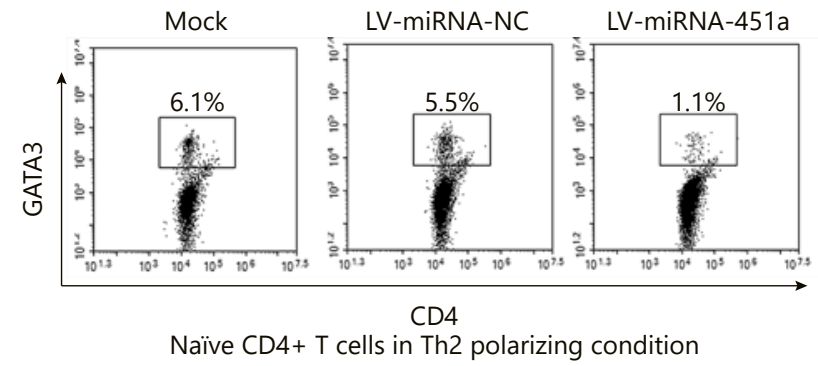

C
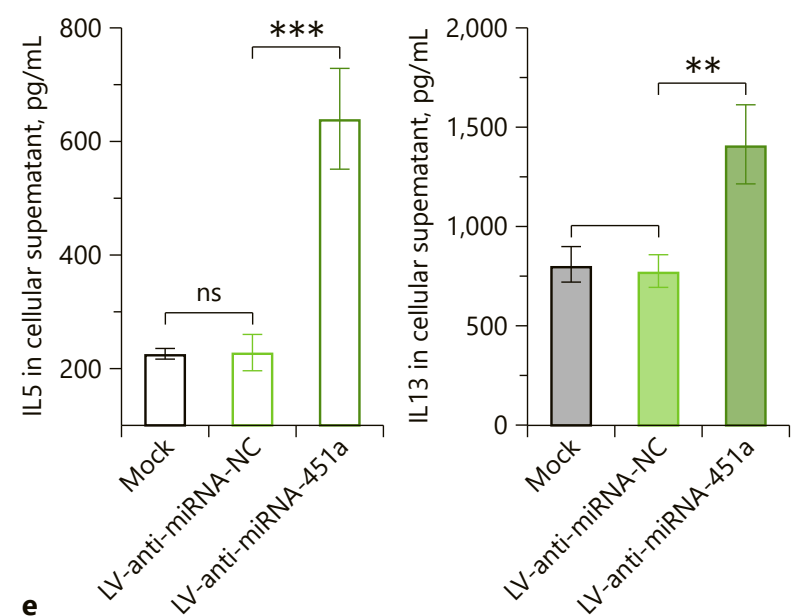

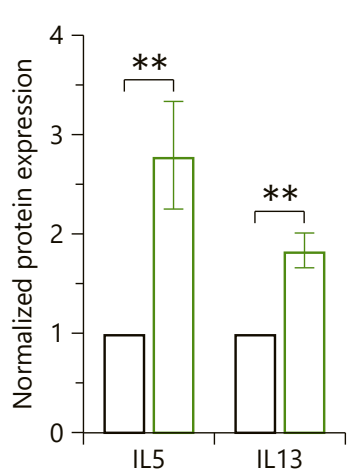

$\square$ LV-anti-miRNA-NC

$\square$ LV-anti-miRNA-451a

b
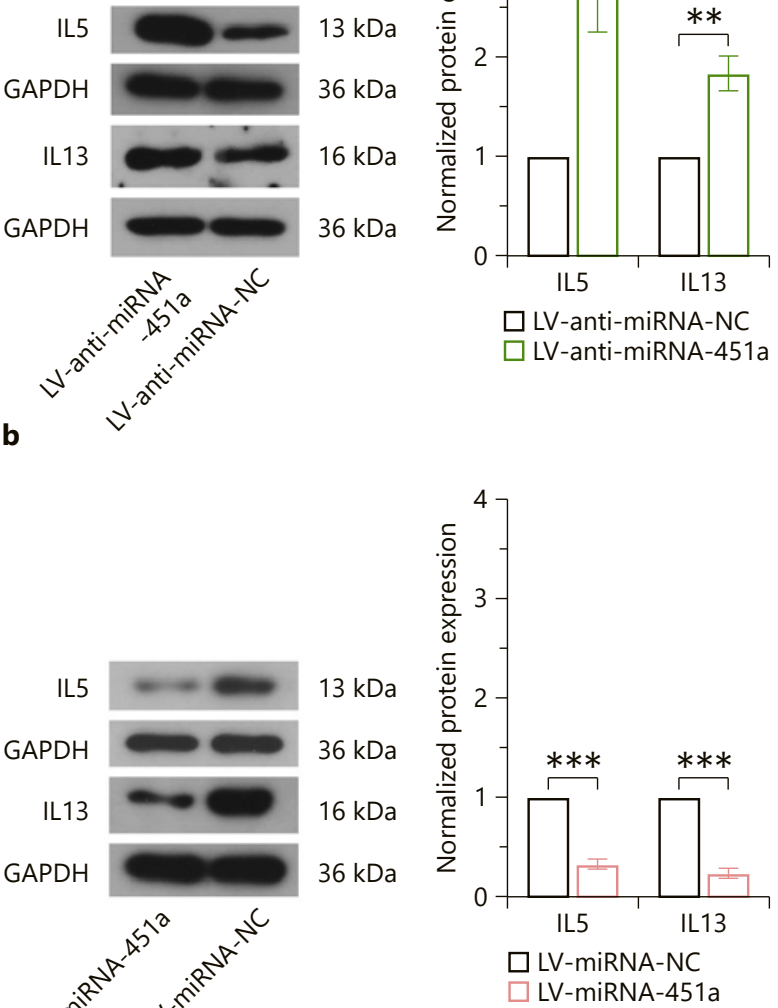

d
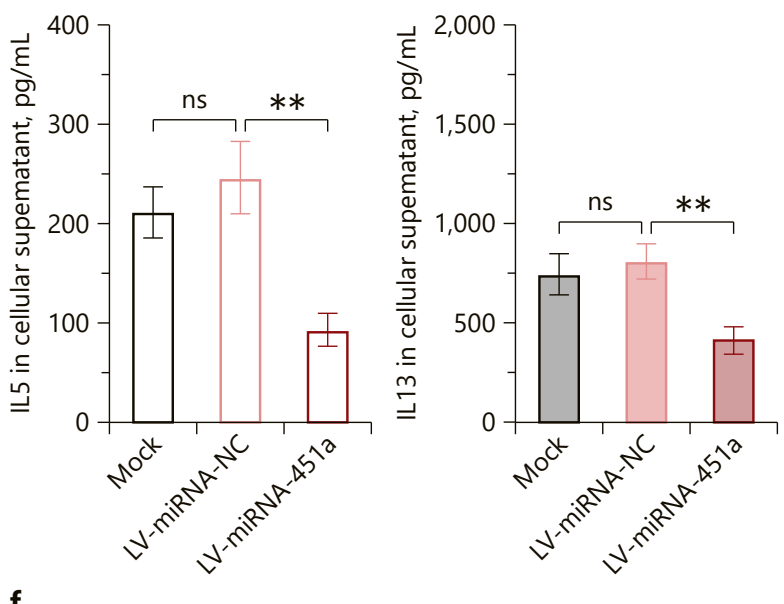

Fig. 3. miRNA-451a suppresses the differentiation of CD4+ T cells towards Th2 cells. a, c Representative images of flow cytometry analysis showing GATA3+CD4+ cells. $\mathbf{b}$, $\mathbf{d}$ The protein levels of IL5 and IL13 in CD4+ T cells detected by Western blot. e, f ELISA analysis showed the level of IL5 and IL13 after silencing or overex- pression of miRNA-451a. Data were analyzed by two-tailed unpaired $t$ test or one-way ANOVA followed by Tukey's multiple comparation $(n=3)$. ${ }^{* *} p<0.01,{ }^{* * *} p<0.001$. ns indicates a $p$ value $>0.05$. 
ETS1_site 1

WT_1 5'...GAGCUGCUCAGGGAGUGUUGGGUGGAACUGUUU...3' miRNA-451a 3'UUGAGUC---AUUACCAUUGCCAAA 5'

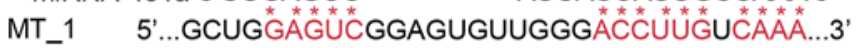

a

ETS1_site 2

WT_2 5'...AGACCAUCCCAGGAAGAGUGGUGGG---UGGUUU...3' miRNA-451a 3'UUGAGUCAUUACCAUUGCCAAA 5' I

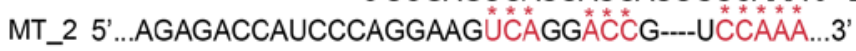

c
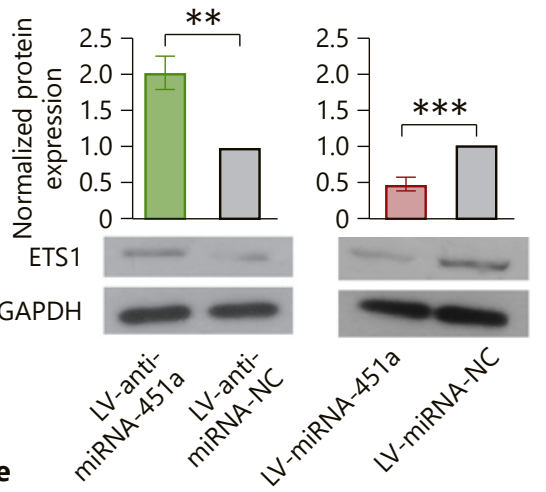
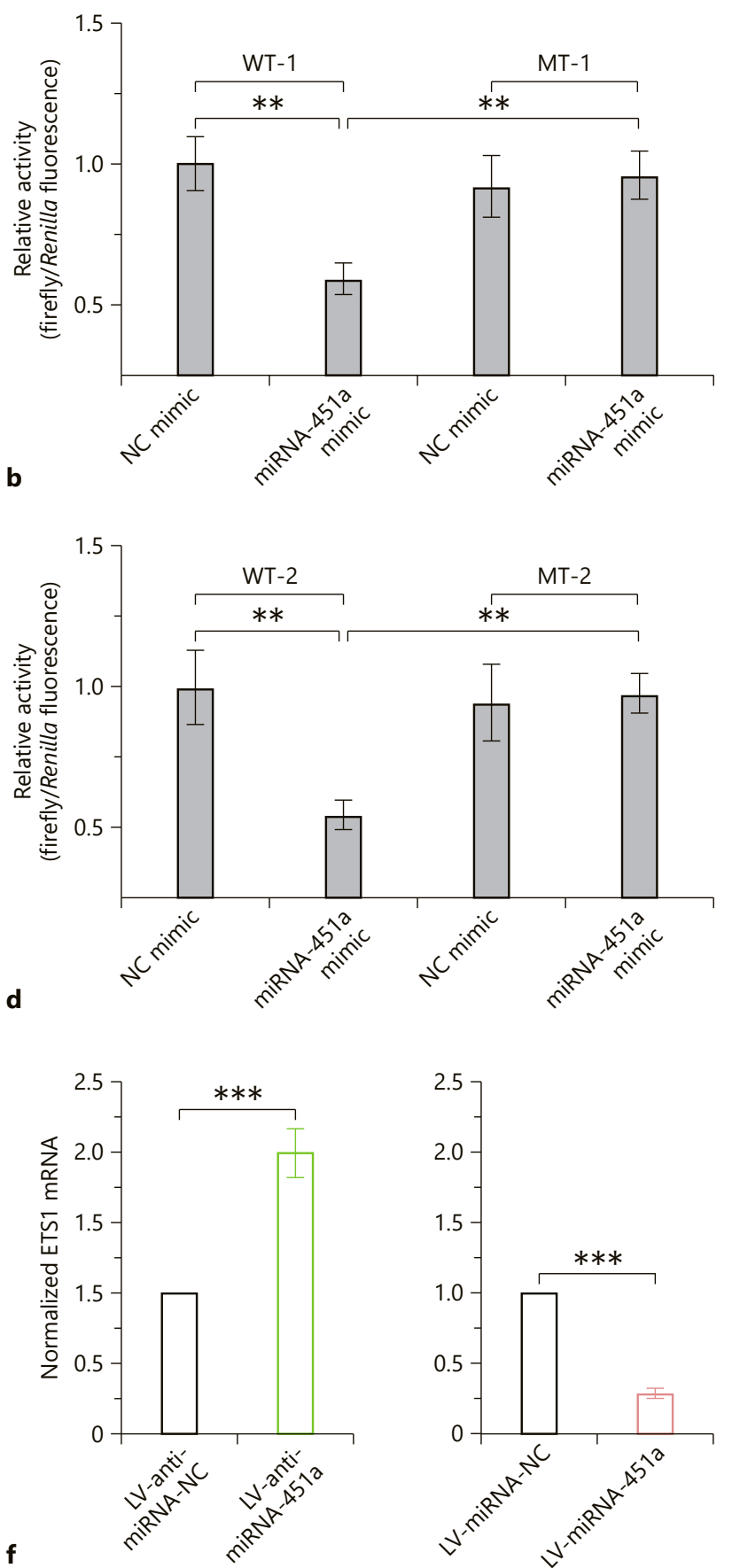

Fig. 4. miRNA-451a directly targets ETS1 in CD4+ T cells in vitro. a, c Miranda (http://www.microrna.org/microrna/home.do) and RNAhybrid (https://bibiserv.cebitec.uni-bielefeld.de/rnahybrid) were used for target prediction. Sequence information of ETS1 WT and mutant-type (MT) 3'UTR. These fragments were inserted into pmirGLO plasmids, and co-transfected into $293 \mathrm{~T}$ cells together with miRNA-451a mimics or NC mimics. b, d The relative luciferase activity was determined and analyzed with one-way ANO-
VA. Additionally, CD4+ T cells were induced with Th2 cell differentiation followed by infection with LV-anti-miRNA-451a and LV-miRNA-451a. The expression levels of ETS1 were analyzed by Western blot (e) and real-time qPCR (f). Data in $\mathbf{e}$ and $\mathbf{f}$ are shown as the mean \pm SD from three separate experiments, with two-tailed unpaired $t$ test $(n=3) .{ }^{* *} p<0.01,{ }^{* * *} p<0.001$. NC, negative control. 


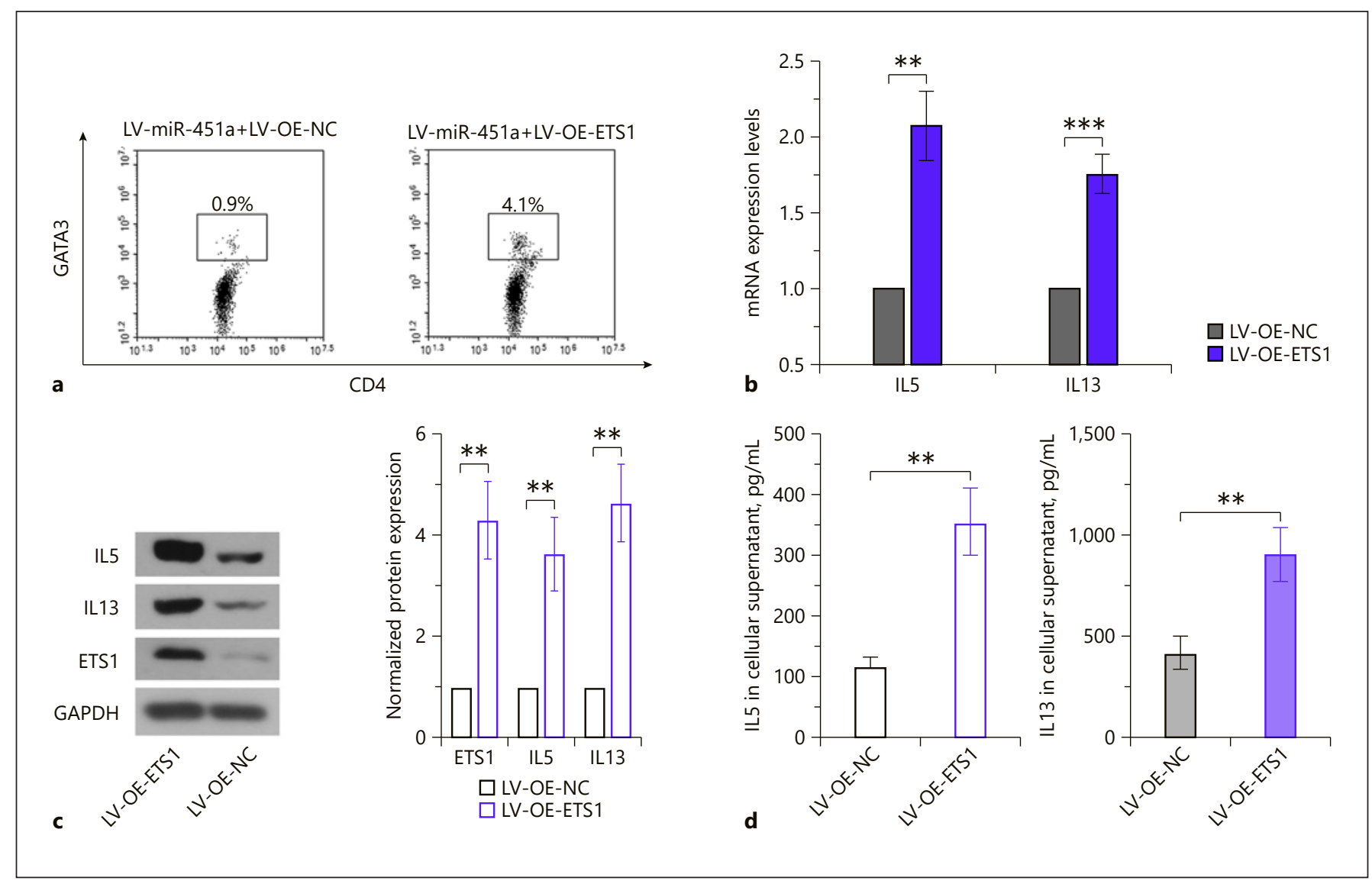

Fig. 5. miRNA-451a inhibits Th2 cell differentiation through downregulating ETS1. Cells overexpressing miRNA-451a were infected with LV-OE-ETS1 or LV-OE-NC. a Representative images of flow cytometry analysis showing GATA3+CD4+ cells. b The mRNA levels of IL5 and IL13 were analyzed by qRT-PCR. c The protein expression of ETS1, IL5, and IL13 were determined with

by real-time qPCR analysis (online suppl. Fig. s3C, D). Interestingly, reduction in the GATA3+CD4+ cell population and production of IL5 and IL13 indicated that miR3646 also inhibited the differentiation of Th2 cells in vitro (online suppl. Fig. s4). Genes that were potential targets for miR-3646 with negative miRNA-gene correlation based on the microarray data are listed in online supplementary Table s2. With the information listed in online supplementary Table s2, their function can be found in GeneCards (https://www.genecards.org/).

\section{miRNA-451a Directly Targets ETS1}

As predicted, miRNA-451a may target ETS1 through two areas on its $3^{\prime} U T R$ (Fig. 4a, c). Dual-luciferase results indicated that the miRNA-451a mimic significantly reduced the relative luciferase activity of ETS1 WT 3'UTR,
Western blot. d ELISA analysis of IL5 and IL13. The quantitative analyses in $\mathbf{b}$ and $\mathbf{c}$ are presented as the mean \pm SD calculated based on three separate experiments with two-tailed unpaired $t$ tests $(n=3) .{ }^{* *} p<0.01,{ }^{* * *} p<0.001$. NC, negative control; OE, overexpression.

but not that of mutant $3^{\prime} \mathrm{UTR}$ (Fig. 4b, d). The inhibitory effects of miRNA-451a on both WT fragments were comparable to each other.

CD4+ T cells in Th2 cell polarization medium were further infected with LV-anti-miRNA-451a or LV-miRNA-451a. Data from real-time qPCR and Western blot demonstrated that ETS1 expression was upregulated following miRNA-451a inhibition, but downregulated when miRNA-451a was overexpressed (Fig. 4e, f). These data together with the dual-luciferase results confirmed ETS1 as a target of miRNA-451a for the first time.

\section{miRNA-451a Inhibits Th2 Cell Differentiation}

through Downregulating ETS1

To determine the role of ETS1 in miRNA-451a-medidated Th2 differentiation, ETS1 was additionally re-ex- 
pressed in cells overexpressing miRNA-451a (online suppl. Fig. s5). The results illustrated that ETS1 re-expression increased the Th2 cell portion and augmented the generation of IL5 and IL13 (Fig. 5a-d). These data demonstrated that miRNA-451a did suppress Th2 cell differentiation by targeting ETS1.

\section{Discussion}

By analyzing the expression of miRNA-451a and ETS1 in CD4+ T cells isolated from lymphocyte samples from healthy and asthmatic children, we first found an opposite expression pattern between them. miRNA-451a expression spontaneously decreased in CD4+ T cells under Th2 induction, whereas ETS1 increased. In vitro, we identified a novel suppressive role of miRNA-451a on Th2 differentiation, in which ETS1 silencing was involved.

The information of miRNA and mRNA expression profiles obtained from microarrays paved the way to identify novel molecules involved in the pathology of pediatric asthma. In agreement with previous studies, our microarray results reported a downregulation of miRNA451a in CD4+ T cells of asthmatic children [19]. Our data further illustrated that miRNA-451a downregulation concurrently occurred with Th2 cell polarization, and that antagonizing its function enhanced the generation of Th2 cytokines, IL5 and IL13. Additional gain-of-function analysis showed that miRNA-451a inhibited the differentiation of Th2 cells. Our work identified for the first time miRNA-451 a as a negative regulator for Th2 polarization. Inconsistent with our findings, Cheng et al. [20] demonstrated that miRNA-451a deficiency led to reduced production of IL4 in mice with systemic lupus erythematosus. Their study suggests that miRNA-451a upregulation, but not its downregulation, is required for Th2 polarization. This phenomenon implies that the role of miRNA451a in regulating the polarization of Th cells may differ in different immune diseases. As a highly conserved miR$\mathrm{NA}$, the sequences of homo, $\mathrm{mmu}$, rno miRNA-451 a were the same (http://mirbase.org). Additional experiments on rodent models are needed to better elucidate the role of miRNA-451a in Th2 polarization in childhood asthma.

A total of 29 upregulated genes were identified as potential targets for miRNA-451a. Among them, ETS1, a molecule that was previously found to be associated with Th2 polarization $[21,16]$, drew our attention. As a transcription factor, ETS1 cooperated with GATA-3 to activate the IL5 promoter in Jurkat cells through a GGAA/T motif [21]. Despite lack of evidence for ETS1 in regulating

miRNA-451a Downregulation

Contributes to Th2 Cell Polarization the activity of the IL13 promoter, a previous study illustrated that ETS1 deletion suppressed IL13 generation in ILC2s [18], suggesting its positive regulatory role in promoting IL13 expression. We analyzed the promoter sequence of IL13 with the JASPER database (http://jaspar. genereg.net/analysis) and found several potential binding sites for ETS1 within its promoter $(<-1,000 \mathrm{bp})$. All the previous evidence suggests ETS1 as a contributor to Th2 cell differentiation. ETS1 expression, in contrast to miRNA-451a, was elevated during IL4-induced Th2 cell expansion in vitro. Additional dual-Luciferase results confirmed ETS1 as a novel target for miRNA-451a. These data together with previous findings suggest that miRNA-451a possibly suppresses Th2 polarization by inhibiting ETS1.

The transcriptional activation effect of ETS1 on IL5/ IL13 has been demonstrated before [21] or is foreseeable (JASPER database). Our present data directly demonstrated that elevating ETS1 expression in CD4+ T cells enhanced the transcription of IL5 and IL13, implying ETS1 may act on their promoter. Previous studies also showed a different functional role of ETS1 than regulating the transcription of Th2 cytokines. Li and Lau [22] demonstrated that the sequential mutation of ETS1 binding sites inhibited the promoter activity of IL10, a maker for regulatory $\mathrm{T}$ (Treg) cells. The immunodeficiency virus trans-activator (Tat) is a strong inducer of IL10 production [23]. ETS1 could further enhance Tat-induced IL10 transcription [22]. In addition, exosomes carrying miRNA-451a promoted Th17 cell differentiation within the tumor microenvironment [24]. These findings along with ours suggest that, under different polarizing conditions for varied types of CD4+ Th cells, ETS1 may selectively bind to the promoter of genes encoding different types of Th cytokines. Further exploration of whether miRNA-451a-ETS1 interaction affects the balance among different types of CD4+ Th cells will help with the growing understanding of molecular and cellular mechanisms of childhood asthma.

Besides ETS1, another 28 genes were identified as potential targets for miRNA-451a. These genes could be annotated into multiple signaling pathways (data not shown). Some of these pathways, including Janus kinasesignal transducer and activator of transcription (JAKSTAT), RAS, and MAPK signaling pathways, have been reported to play an important role in orchestrating type 2 allergic inflammation [25-27]. Such results inspire us to further explore the cellular mechanisms underlying miRNA-451a-mediated Th2 polarization in pediatric asthma. 
In conclusion, we have demonstrated that miRNA451a downregulation and the subsequent ETS1 elevation contribute to the type 2 allergic inflammatory response. Our work reveals the deregulated miRNA-451a-ETS1 axis as a novel molecule mechanism that underlies the pathogenesis of childhood asthma.

\section{Statement of Ethics}

The informed consents of asthmatic and healthy children were provided by their legal guardians. The whole study was approved by China Medical University, and was conducted ethically in accordance with the World Medical Association Declaration of Helsinki.

\section{Conflict of Interest Statement}

The authors have no conflicts of interest to declare.

\section{Funding Sources}

There were no sources of funding in relation to this work.

\section{Author Contributions}

T.W. and Y.S.: conception of the study. T.W.: designed and carried out the experiments, and drafted the manuscript. T.W. and Q.Z.: analyzed the data. All authors approved the final version and submission of the manuscript.

\section{References}

1 Fahy JV. Type 2 inflammation in asthma present in most, absent in many. Nat Rev Immunol. 2015 Jan;15(1):57-65.

2 Anderson GP. Endotyping asthma: new insights into key pathogenic mechanisms in a complex, heterogeneous disease. Lancet. 2008 Sep;372(9643):1107-19.

3 Wenzel SE. Asthma phenotypes: the evolution from clinical to molecular approaches. Nat Med. 2012 May;18(5):716-25.

4 Locksley RM. Asthma and allergic inflammation. Cell. 2010 Mar;140(6):777-83.

5 Holgate ST. Innate and adaptive immune responses in asthma. Nat Med. 2012 May;18(5): 673-83.

6 Halim TY, Steer CA, Mathä L, Gold MJ, Martinez-Gonzalez I, McNagny KM, et al. Group 2 innate lymphoid cells are critical for the initiation of adaptive T helper 2 cell-mediated allergic lung inflammation. Immunity. 2014 Mar;40(3):425-35.

7 Moldaver DM, Larché M, Rudulier CD. An Update on Lymphocyte Subtypes in Asthma and Airway Disease. Chest. 2017 May;151(5): 1122-30.

8 Bartemes KR, Kephart GM, Fox SJ, Kita H. Enhanced innate type 2 immune response in peripheral blood from patients with asthma. J Allergy Clin Immunol. 2014 Sep;134(3):671678.e4.

9 Smith SG, Chen R, Kjarsgaard M, Huang C, Oliveria JP, O'Byrne PM, et al. Increased numbers of activated group 2 innate lymphoid cells in the airways of patients with severe asthma and persistent airway eosinophilia. J Allergy Clin Immunol. 2016 Jan;137(1): 75-86.e8.

10 Adcock IM, Mumby S. Glucocorticoids. Handb Exp Pharmacol. 2017;237:171-96.

11 Farne HA, Wilson A, Powell C, Bax L, Milan SJ. Anti-IL5 therapies for asthma. Cochrane Database Syst Rev. 2017 Sep;9:CD010834.
12 Yang M, Mattes J. Discovery, biology and therapeutic potential of RNA interference, microRNA and antagomirs. Pharmacol Ther. 2008 Jan;117(1):94-104

13 Malmhall C, Alawieh S, Lu Y, Sjostrand M, Bossios A, Eldh M, et al. MicroRNA-155 is essential for $\mathrm{T}(\mathrm{H}) 2$-mediated allergen-induced eosinophilic inflammation in the lung. $\mathrm{J} \mathrm{Al}-$ lergy Clin Immunol. 2014 May;133(5):142938.e1-7.

14 Negi V, Paul D, Das S, Bajpai P, Singh S, Mukhopadhyay A, et al. Altered expression and editing of miRNA-100 regulates iTreg differentiation. Nucleic Acids Res. 2015 Sep;43(16): 8057-65.

15 Alharris E, Alghetaa H, Seth R, Chatterjee S, Singh NP, Nagarkatti M, et al. Resveratrol attenuates allergic asthma and associated inflammation in the lungs through regulation of miRNA-34a that targets FoxP3 in mice. Front Immunol. 2018 Dec;9:2992.

16 Zeng Z, Wang K, Li Y, Xia N, Nie S, Lv B, et al. Down-regulation of microRNA-451a facilitates the activation and proliferation of CD4+ $\mathrm{T}$ cells by targeting Myc in patients with dilated cardiomyopathy. J Biol Chem. 2017 Apr;292(14):6004-13.

17 Findlay VJ, LaRue AC, Turner DP, Watson PM, Watson DK. Understanding the role of ETS-mediated gene regulation in complex biological processes. Adv Cancer Res. 2013;119: 1-61.

18 Zook EC, Ramirez K, Guo X, van der Voort G, Sigvardsson M, Svensson EC, et al. The ETS1 transcription factor is required for the development and cytokine-induced expansion of ILC2. J Exp Med. 2016 May;213(5): 687-96.

19 Liu F, Qin HB, Xu B, Zhou H, Zhao DY. Profiling of miRNAs in pediatric asthma: upregulation of miRNA-221 and miRNA-485-3p. Mol Med Rep. 2012 Nov;6(5):1178-82.
20 Cheng J, Wu R, Long L, Su J, Liu J, Wu XD, et al. miRNA-451a Targets IFN Regulatory Factor 8 for the Progression of Systemic Lupus Erythematosus. Inflammation. 2017 Apr; 40(2):676-87.

21 Blumenthal SG, Aichele G, Wirth T, Czernilofsky AP, Nordheim A, Dittmer J. Regulation of the human interleukin-5 promoter by Ets transcription factors. ETS1 and ETS2, but not ELF-1, cooperate with GATA3 and HTLV-I TAX1. J Biol Chem. 1999 Apr; 274(18):12910-6.

$22 \mathrm{Li} \mathrm{JC}$, Lau AS. A role for mitogen-activated protein kinase and Ets-1 in the induction of interleukin-10 transcription by human immunodeficiency virus-1 Tat. Immunology. 2007 Jul;121(3):337-48.

23 Breen EC. Pro- and anti-inflammatory cytokines in human immunodeficiency virus infection and acquired immunodeficiency syndrome. Pharmacol Ther. 2002 Sep;95(3):295304.

24 Liu F, Bu Z, Zhao F, Xiao D. Increased T-helper 17 cell differentiation mediated by exosome-mediated microRNA-451 redistribution in gastric cancer infiltrated T cells. Cancer Sci. 2018 Jan;109(1):65-73.

25 Pernis AB, Rothman PB. JAK-STAT signaling in asthma. J Clin Invest. 2002 May;109(10): 1279-83.

26 Khorasanizadeh M, Eskian M, Gelfand EW, Rezaei N. Mitogen-activated protein kinases as therapeutic targets for asthma. Pharmacol Ther. 2017 Jun;174:112-26.

27 Bratt JM, Chang KY, Rabowsky M, Franzi LM, Ott SP, Filosto S, et al. Farnesyltransferase inhibition exacerbates eosinophilic inflammation and airway hyperreactivity in mice with experimental asthma: the complex roles of Ras GTPase and farnesylpyrophosphate in type 2 allergic inflammation. J Immunol. 2018 Jun;200(11):3840-56. 